\title{
Monoenergetic ion beams from ultrathin foils irradiated by ultrahigh-contrast circularly polarized laser pulses
}

\author{
O. Klimo, ${ }^{*}$ J. Psikal, and J. Limpouch \\ Faculty of Nuclear Sciences and Physical Engineering, Czech Technical University in Prague, Brehova 7, 11519 Praha 1, \\ Czech Republic
}

V.T. Tikhonchuk

Centre Lasers Intenses et Applications, Université Bordeaux 1-CNRS-CEA, 33405 Talence Cedex, France

(Received 3 September 2007; published 17 March 2008)

\begin{abstract}
Acceleration of ions from ultrathin foils irradiated by intense circularly polarized laser pulses is investigated using one- and two-dimensional particle simulations. A circularly polarized laser wave heats the electrons much less efficiently than the wave of linear polarization and the ion acceleration process takes place on the front side of the foil. The ballistic evolution of the foil becomes important after all ions contained in the foil have been accelerated. In the ongoing acceleration process, the whole foil is accelerated as a dense compact bunch of quasineutral plasma implying that the energy spectrum of ions is quasimonoenergetic. Because of the ballistic evolution, the velocity spread of an accelerated ion beam is conserved while the average velocity of ions may be further increased. This offers the possibility to control the parameters of the accelerated ion beam. The ion acceleration process is described by the momentum transfer from the laser beam to the foil and it might be fairly efficient in terms of the energy transferred to the heavy ions even if the foil contains a comparable number of light ions or some surface contaminants. Two-dimensional simulations confirm the formation of the quasimonoenergetic spectrum of ions and relatively good collimation of the ion bunch, however the spatial distribution of the laser intensity poses constraints on the maximum velocity of the ion beam. The present ion acceleration mechanism might be suitable for obtaining a dense high energy beam of quasimonoenergetic heavy ions which can be subsequently applied in nuclear physics experiments. Our simulations are complemented by a simple theoretical model which provides the insights on how to control the energy, number, and energy spread of accelerated ions.
\end{abstract}

DOI: 10.1103/PhysRevSTAB.11.031301

PACS numbers: 52.38.Kd, 52.50.Jm, 52.65.Rr, 52.40.Kh

\section{INTRODUCTION}

Collimated beams of energetic ions produced in interactions of short intense laser pulses with solid density thin foil targets have been demonstrated in experiments [1-4] and particle simulations [5]. These ion beams are attracting much attention due to a wide range of their potential applications covering high resolution radiography imaging, probing for strong electrostatic fields induced in plasmas, radioisotope production, isochoric heating, cancer therapy etc. [6]. Many of these applications require a narrow (quasimonoenergetic) energy spectrum of accelerated ions with a relatively low energy spread. The depthdose distributions of energy deposited by such ion beams exhibit a strong Bragg peak at the end of the penetration range and thus they can heat a relatively small and welldefined volume inside the target to high temperatures.

The energy distribution of energetic ions obtained in the current experiments is usually exponential with almost $100 \%$ energy spread up to a cutoff energy [7]. A tailoring of the energy distribution of ions has been demonstrated via the target material composition $[8,9]$. In this case, a quasimonoenergetic spectrum of light ions may be pro-

\footnotetext{
*klimo@watt.fjfi.cvut.cz
}

duced from their interaction with the fastest heavy ions. However, a significant fraction of the absorbed laser energy is contained in the heavy ion bunch and thus acceleration of light ions is not really efficient. Moreover, only the light ions demonstrate a remarkable peak in the energy spectrum and this process is not suitable for acceleration of monoenergetic bunches of heavy ions.

In this paper, an alternative and a fairly efficient mechanism capable of producing monoenergetic bunches of ions of varies species is presented and studied using the particle-in-cell (PIC) simulations. This ion acceleration mechanism takes place in the radiation pressure dominant regime. The laser beam pushes electrons from the front surface into the target via the ponderomotive force. A strong positive quasistatic electric field is induced due to the resulting charge separation and through this field the momentum acquired by electrons from the laser beam is efficiently transferred to ions. We find that the momentum transfer is maximized when an ultrahigh-contrast circularly polarized laser wave is normally incident on the surface of an overdense foil. In this case most electron heating mechanisms (resonance absorption, vacuum heating, heating by the oscillating ponderomotive force) are inefficient and the laser beam is largely reflected. The acceleration process is accompanied by the ballistic evo- 
lution of the target itself. Because of this evolution, the momentum delivered by the laser pulse is distributed among the target ions so that most of them attain approximately the same velocity in the laser propagation direction. Therefore, it is possible to accelerate the whole foil as a compact dense block of quasineutral plasma. An ultrathin foil accelerated this way can be regarded as a plasma mirror copropagating with the laser pulse like in [10]. Moreover, the presented mechanism enables one to control both the energy and the number of ions contained in the monoenergetic bunch.

Acceleration of ions from ultrathin foils has been theoretically studied in several recent papers [11-13] for similar laser-target interaction conditions. The main difference in our approach is in utilization of a circular laser polarization that inhibits a strong electron heating. Energetic electrons are really undesirable in our approach as they may cause a premature explosion of the foil, whereas it is necessary to confine the ions in a relatively small volume for their efficient acceleration. The interaction of a circularly polarized laser beam with a dense target has been studied by Macchi et al. [14] and we will develop the ideas presented in their paper. We consider a thinner foil and a longer or more energetic laser pulse. This enables us to go a step further and to describe an acceleration process after the whole target is set in motion.

This paper presents the results of our PIC simulations and their interpretations based on a simple analytical model. Comparison with the experimental results is not provided. To our knowledge, the only experiment of ion acceleration with the circularly polarized laser pulse has been published in [15]. However, the parameters of the laser pulse and of the foil used in [15] do not fall into the range of applicability of our ion acceleration model as the foil is too thick and dense and the laser pulse is not enough energetic to set the whole foil into motion. Nevertheless, the results of this experiment confirm the crucial point of our ion acceleration model, namely, that the efficiency of electron heating is largely suppressed when a high-contrast circularly polarized laser pulse is used in comparison with the linearly polarized pulse.

The paper is organized as follows. Our numerical models based on the 1D3V and 2D3V PIC simulation codes are presented in Sec. II. Section III A describes the theory of the ion acceleration process and introduces the basic principles of our ion acceleration mechanism. Ions are accelerated, in particular, in the forward laser incidence direction in the case of this mechanism. Therefore, the backward acceleration of ions will not be discussed here. The role of electron heating and the influence of the laser wave polarization are of prime importance and they are described in Sec. III B. The results of our 1D3V PIC simulations are presented and discussed in detail in Sec. III C. It is explained how the monoenergetic distribution of the accelerated ions emerges naturally in this case. Our results are accompanied by a simple analytical model which provides the basic insight into the ion acceleration mechanism and which can be used for reasonably accurate estimates. The simulations of a multiple species target are presented and discussed in Sec. IIID. The effects related to the second, lateral dimension are studied in Sec. III E by using the 2D3V PIC simulation code. The main results and ideas presented in this paper are summarized in Sec. IV together with our concluding remarks.

\section{PARTICLE-IN-CELL SIMULATION MODELS}

Numerical simulations of the laser beam interaction with a foil target and of the process of ion acceleration are performed using one- and two-dimensional particle-incell (PIC) codes. Both codes are relativistic and electromagnetic and they take into account all three components of particle velocities and electric and magnetic fields (1D3V and 2D3V). The one-dimensional PIC code [16] evolved from the code LPIC + + [17]. It includes elastic collisions and variable plasma ionization, but for computational reasons neither of these features is employed in the simulations presented in this paper. To resolve the processes taking place during the interaction with enough accuracy and to avoid significant numerical noise, the size of the computational cell in one-dimensional simulation is smaller than $0.3 \mathrm{~nm}$ and each cell is occupied by more than 600 particles. The simulation box is always sufficiently large so that no particles can reach the boundaries during the simulation time.

The two-dimensional simulations have been performed with our newly developed collisionless PIC code [18] which is parallelized via OpenMP scheme. In this code, the relativistic equations of motion are solved by the twostep Boris rotation and the fields in the positions of particles are determined by bilinear interpolation. The zigzag scheme [19] is employed for calculation of current densities in order to guarantee an automatic compliance with the continuity equation. The boundary conditions for particles are absorbing, particles reaching the boundaries of the simulation box are frozen. For the solution of Maxwell's equations, damping layers are added near the simulation box boundaries in order to eliminate spurious reflection of outgoing electromagnetic waves. The corresponding algorithm described for the one-dimensional geometry in Ref. [20] was successfully adopted for twodimensional case in our code.

\section{THEORETICAL MODEL AND THE SIMULATION RESULTS}

\section{A. Ion acceleration process - basic principles}

In the interaction of a short intense laser pulse with an overdense foil target, the ions are accelerated by the quasistatic charge-separation fields induced on both target surfaces, at the front and the rear sides. The electrostatic field 
on the front side of the target is generated by fast electrons accelerated into the target. This field gives rise to the return current and also to acceleration of ions toward the target interior. Moreover, the fast electrons penetrating through the target create an electrostatic field at the rear surface of the foil which is responsible for acceleration of ions into the vacuum. The latter process is known as the target normal sheath acceleration (TNSA) [5].

It has been demonstrated [21] that acceleration of ions at the rear surface is more efficient and it is responsible for the highest energy and best collimated ion beams in the conditions of many recent experiments. This is, in particular, due to a steep plasma density profile on the rear surface of the target which remains undisturbed until the energetic electrons arrive there. In consequence, the self-induced rear side quasistatic electric field can be very strong and concentrated in a very narrow region. On the other hand, the density profile on the front surface of the target, which is formed due to absorption of the laser prepulse, is usually relatively long, the electrostatic field extends over a wider spatial region, and it is weaker. Moreover, the front surface is bent by the ponderomotive force of the laser pulse during the interaction and thus the accelerated ions may have a relatively large angular spread. The formation of preplasma on the laser side of the target has been suppressed in recent experiments [22,23] where the contrast of laser pulse was improved by using the plasma mirrors. In this case, acceleration of ions from the front surface of the target may also be efficient. The fast electron circulation across the foil makes a little difference in front and rear side acceleration in ultrathin targets in this case.

The ion acceleration process described in this paper makes use of ultrathin foils and ultrahigh-contrast laser pulses as well. However, the essential requirements of the presented ion acceleration mechanism are the suppression of the electron heating and enhancement of the front side acceleration (FSA) process via the laser ponderomotive force. Compliance to these conditions enables one to accelerate the target as a compact block of a quasineutral plasma. This is achieved by a combination of the FSA process and the ballistic evolution of the target itself. Because of the localization of the ponderomotive force on the front side, all ions of the target are successively crossing the acceleration zone and gaining approximately the same velocity. Moreover, the slower and heavier ions are stronger accelerated since they are affected for a longer time by the force. This naturally results in acceleration of the whole target to a well-defined macroscopic propagation velocity. The relatively cold electrons are enabling a good screening of the electrostatic field and thus preventing the accelerated ions from the Coulomb explosion.

\section{B. Fast electrons-influence of the laser polarization}

The role of fast electrons is very important in most of the recently proposed ion acceleration mechanisms [5,13,21].
Here, the term "fast electrons" stands especially for those relativistic electrons, which are energetic enough to easily cross the potential barrier formed by the quasistatic chargeseparation field at the front side of the target and to penetrate the foil subsequently. These electrons contribute to the electrostatic field at the front surface, which is responsible for the FSA process. However, in a steep plasma density profile the fast electron density is much less than the plasma density, and the electrostatic chargeseparation field may be largely screened by the return current cold electrons in this region. Thus, the contribution of fast electrons to the FSA process is not essential. Consequently, the generation of fast electrons should be suppressed in order to inhibit the rear side acceleration.

It was demonstrated in Refs. $[14,24]$ and confirmed also in our simulations, that the strong electron heating is suppressed in a circularly polarized laser wave if it is incident normally onto a very steep overdense plasma. This is a direct consequence of the absence of the component of the laser electric field perpendicular to the target surface, the absence of the oscillating part of the ponderomotive force, and the absence of the underdense plasma. This allows one to avoid the well-known electron acceleration processes such as the ponderomotive acceleration by the oscillating $\mathbf{j} \times \mathbf{B}$ force, the resonance absorption, the vacuum heating, and the parametric instabilities. The laser pulse energy is largely reflected, at least in the initial stage of the interaction, and the laser pulse acts on the target surface as a piston by the force of radiation pressure.

There is also another reason for avoiding the electron heating. As the foil considered in this ion acceleration concept should be very thin, the fast electrons circulate freely across the target and create the electrostatic field almost symmetrically on the front and rear surfaces. This produces the target expansion and an initially ultrathin foil may explode before the end of the acceleration process and become transparent for the laser light. This is a selfenhancing effect, as the scale length of the density profile on the front side of the target increases, the laser pulse penetrates deeper and generation of fast electrons becomes more and more efficient during the foil expansion.

The difference between the circular polarization (CP) and the linear polarization (LP) of the laser wave interacting with an ultrathin foil is clearly seen in Figs. 1 and 2. These figures result from one-dimensional (1D) PIC simulations where a $100 \mathrm{fs}$ long ( $\sin ^{2}$ temporal envelope) laser pulse with the wavelength $\lambda=800 \mathrm{~nm}$ and the maximum intensity of $1.5 \times 10^{20} \mathrm{~W} / \mathrm{cm}^{2}$ is normally incident (from the left) on the foil. The density of ions in the foil is $3.5 \times$ $10^{22} \mathrm{~cm}^{-3}$ and the initial thickness of the foil is $32 \mathrm{~nm}$. The foil has a steplike density profile on both surfaces and it is composed of cold fully ionized carbon ions and electrons with initial temperature $T_{0}=1 \mathrm{keV}$.

In the case of CP pulse shown in Fig. 1(a), the energy distribution of electrons follows approximately a two- 

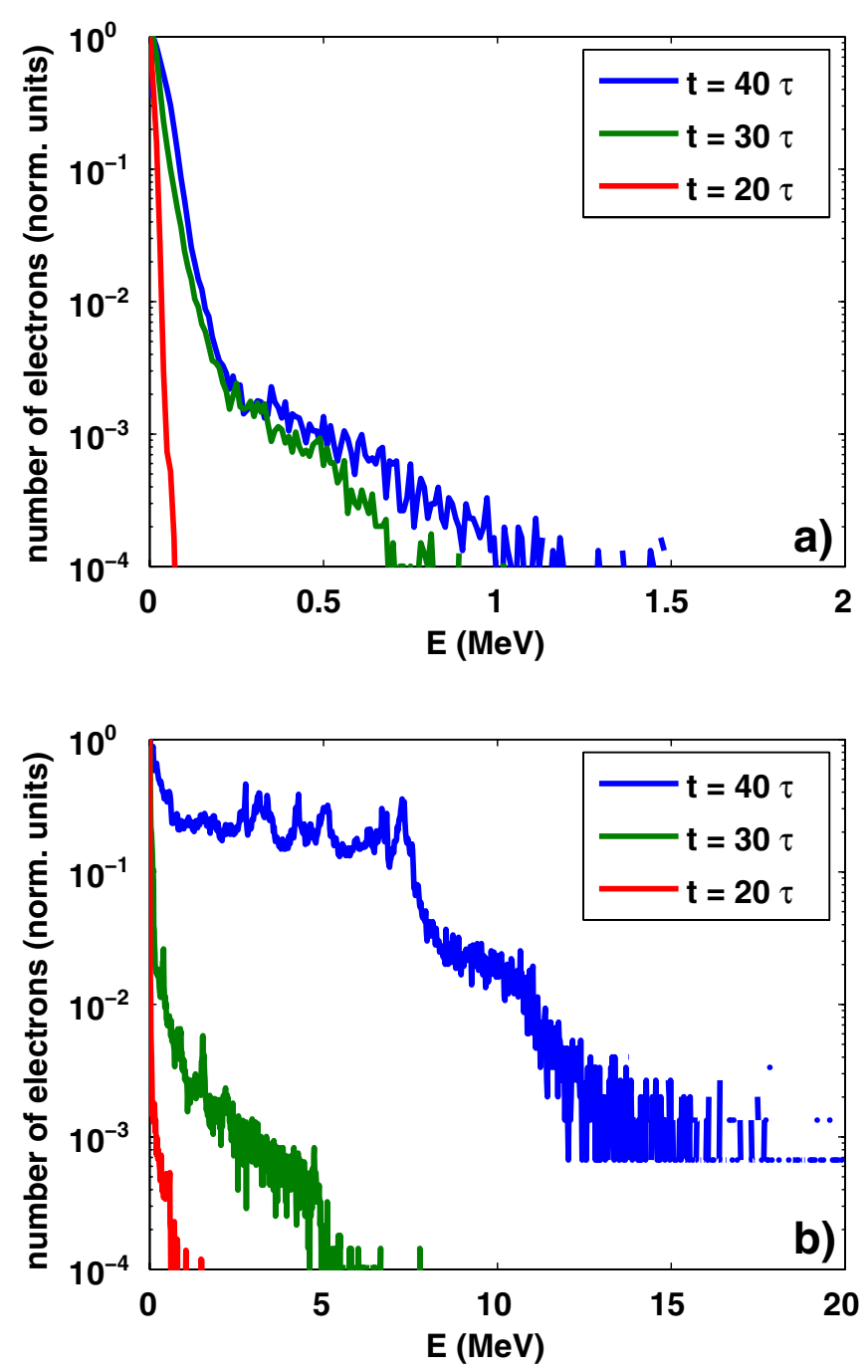

FIG. 1. (Color) Temporal evolution of the electron energy distributions in the 1D3V PIC simulation during the interaction of circularly (a) and linearly (b) polarized relativistic $\left(I_{L}=1.5 \times\right.$ $\left.10^{20} \mathrm{~W} / \mathrm{cm}^{2}\right)$ laser pulse with ultrathin $(32 \mathrm{~nm})$ overdense $\left(n_{e}=\right.$ $2.1 \times 10^{23} \mathrm{~cm}^{-3}$ ) foil. The distributions contain all simulation electrons and they are normalized to have the same maximum values. The laser pulse is $100 \mathrm{fs}$ long ( $\sin ^{2}$ temporal envelope) and the time is measured in laser periods $\tau$. At the time $40 \tau$ (maximum intensity on the target), the foil irradiated by the linearly polarized laser pulse becomes transparent to the laser pulse. Note the different scales on abscissas.

temperature Maxwellian function. The higher temperature corresponds to the electrons located in the laser-target interaction region, whereas the lower temperature corresponds to the bulk electrons which are deeper inside the target. The temperatures of both components change only weakly around the laser pulse maximum. In the case of the LP pulse shown in Fig. 1(b), the temperature of electrons is much higher and, moreover, it rises many times during the interaction. Almost all electrons are relativistic around the laser pulse maximum ( $t=40 \tau$, where $\tau=\lambda / c$ is the laser wave period) in this case and the foil is already underdense
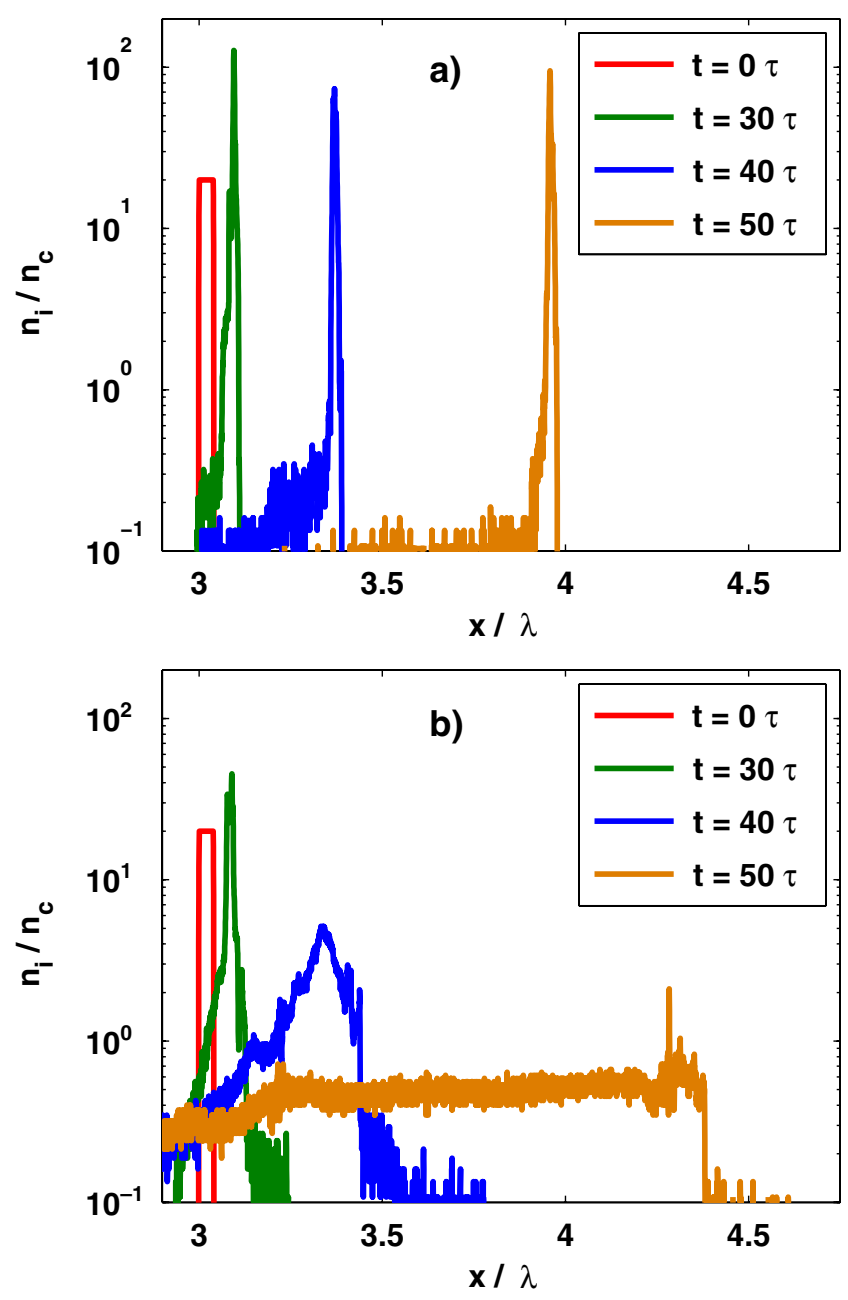

FIG. 2. (Color) Temporal evolution of the ion density profiles obtained in the same simulation as in Fig. 1. The foil irradiated by the $\mathrm{CP}$ laser pulse propagates in the laser incidence direction and it conserves its initial volume [panel (a)]. The foil irradiated by the LP laser pulse explodes [panel (b)].

to the relativistic laser pulse at this time (as can be seen in Fig. 2). Thus, the electrons have also the velocity component associated with oscillations in the field of the laser wave.

Considering the difference between electron heating with CP and LP laser pulse, it is also possible to argue by the motion of a single electron in the field of the laser wave and the plasma field. In the case of the CP laser pulse, electrons are confined in the laser-target interaction region by the quasistatic electric field induced in plasma, as will be demonstrated in the next subsection. On the front surface of the region where electrons are compressed, the smoothly changing ponderomotive force of the laser pulse is balanced by the electrostatic force and the motion of electrons in the target normal direction is negligible. Thus, the electron trajectory is smoothly circular in the plane parallel with the target surface and the kinetic energy of electrons depends, in particular, on the laser pulse ampli- 


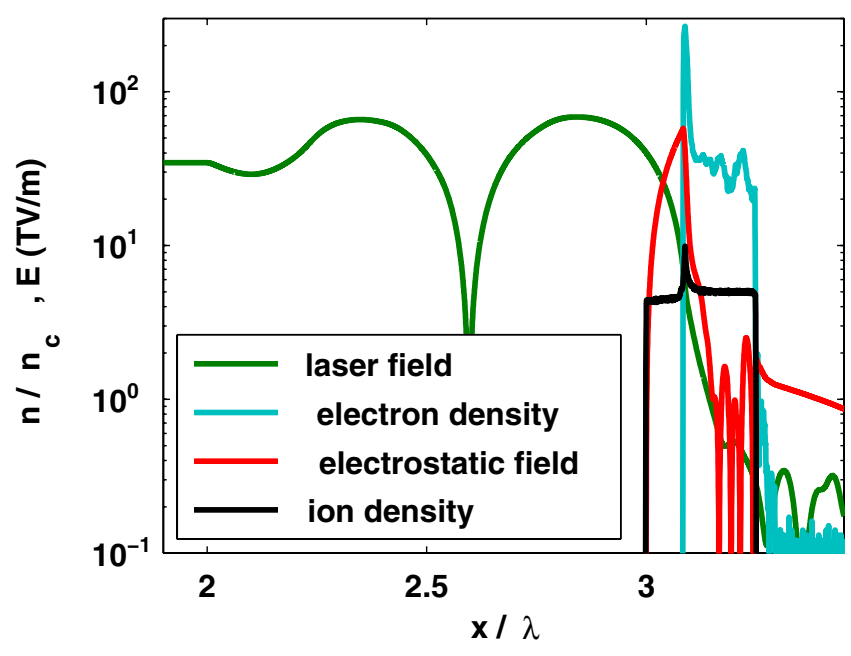

FIG. 3. (Color) Snapshot of the electric field, $|\vec{E}|$, and the densities of electrons and ions taken from the 1D3V PIC simulation. The 80 fs long (a rectangular temporal envelope) CP laser pulse with the wavelength of $800 \mathrm{~nm}$ and the intensity of $3.5 \times$ $10^{20} \mathrm{~W} / \mathrm{cm}^{2}$ is normally incident (from the left) on the ultrathin $(200 \mathrm{~nm})$ overdense $\left(n_{e}=5.25 \times 10^{22} \mathrm{~cm}^{-3}\right)$ fully ionized carbon foil. The time is one laser period after the beginning of the interaction. The densities are normalized to the critical density.

tude. On the contrary, the motion of electrons in the case of the LP laser pulse may be very rough as the ponderomotive force drives their oscillations across the target surface and their energy may change between the maximum and zero at twice the laser period.

Figure 2 shows that the whole foil is accelerated in the laser propagation direction in both cases at the early stage of the interaction, $t<30 \tau$. Nevertheless, near the laser pulse maximum, the foil irradiated by the LP wave explodes in both directions due to a very high electron temperature, whereas the foil irradiated by the $\mathrm{CP}$ wave still conserves its initial volume. The same behavior as the one observed in our PIC simulations with CP laser pulse, Fig. 2(a), has also been presented in [25] (in Fig. 3). That paper is however concerned with electron compression by the CP laser pulse inside an ultrathin foil and it does not discuss this behavior in the context of ion acceleration.

Further simulations presented in the next section provide more details of the ion acceleration process driven by the CP laser pulse.

\section{Circular polarization-formation of a monoenergetic ion spectrum}

The 1D3V PIC simulation results included in this subsection rely on the following parameters. A circularly polarized laser wave with the wavelength $\lambda=800 \mathrm{~nm}$ and the intensity $I_{L}=3 \times 10^{20} \mathrm{~W} / \mathrm{cm}^{2}$ is normally incident (from the left) onto the front surface of the foil. The laser pulse is $80 \mathrm{fs}$ long $(30 \tau)$ and it has a rectangular temporal envelope. This temporal profile is chosen to simplify the analytical estimates that accompany our simu- lation results. This has no major effect on the ion acceleration process. It behaves the same way as it is shown in Fig. 2. The foil used in our simulation is $200 \mathrm{~nm}$ thick $(0.25 \lambda)$ and it is composed of cold fully ionized carbon ions of the density $8.75 \times 10^{21} \mathrm{~cm}^{-3}$. The initial electron temperature is $T_{0}=1 \mathrm{keV}$. The foil has steplike density profiles on both surfaces and it is initially located $3 \lambda$ from the left boundary of the simulation box.

The laser-target interaction can be divided into several subsequent steps, each of them taking place on a different time scale. The first step is characterized by a very rapid response of electrons to the electromagnetic field of the laser wave. Namely, the ponderomotive force pushes all electrons from the target surface deeper into the target. This stage is very short and it lasts only until a quasisteady state is established where the radiation pressure is balanced by the electrostatic pressure. This pressure balance is also included in the ion acceleration model of Macchi et al. [14]. Amplitude of the electrostatic field on the front surface of the cloud of compressed electrons is consequently given by the relation

$$
\frac{1}{2} \varepsilon_{0} E_{\mathrm{es}}^{2}=\frac{1+R}{c} I_{L}
$$

where $I_{L}$ is the laser pulse intensity, $c$ is the velocity of light, and $R$ is the target reflectivity. (We assume $R \simeq 1$ in the following estimates.) According to Eq. (1), the electrostatic field has the same amplitude as the laser field. This is confirmed by the snapshot shown in Fig. 3 which was taken from the PIC simulation just one laser period after the beginning of interaction. The amplitudes of both the electrostatic and the laser electric field are presented there. The incident laser electric field amplitude should be constant in the case of a circular polarization and a rectangular temporal profile. The oscillating structure observed in Fig. 3 in the front of the target is due to the interference of the incident and the reflected laser waves. This interference results from the very fast (adiabatic) compression of electrons by the radiation pressure and it is not so significant if the laser pulse has a smooth temporal envelope.

The amplitude of the incident laser field, $34 \mathrm{TV} / \mathrm{m}$, can be deduced from the plateau before the spatial position $2 \lambda$ in Fig. 3. This is comparable to the electrostatic field amplitude $57.5 \mathrm{TV} / \mathrm{m}$. The structure of the electrostatic field conforms with our essential requirement of the dominance of the FSA process.

As the equilibrium state is established, the compressed electrons do not further propagate deeper into the target because they are confined by the strong electrostatic field in a thin layer. (This layer is denoted as compressed electron cloud in the following.) As the electrons are expelled from the laser field, their heating is suppressed. Therefore, the whole population of electrons in the compressed electron cloud has a single temperature and they 
form the higher temperature Maxwellian part in the energy distribution shown in Fig. 1(a).

The electrostatic field in the electron depletion layer is defined by the space charge separation. Its amplitude can be calculated from the Poisson equation as

$$
E_{\mathrm{es}}(l)=e n_{e 0} l / \varepsilon_{0},
$$

where $n_{e 0}$ is the initial free electron density in the target and $l$ is the thickness of the electron depletion layer. Combining the Poisson equation with the pressure balance given in Eq. (1), one can estimate $l$ as follows:

$$
l=\sqrt{\frac{4 I_{L} \varepsilon_{0}}{c n_{e 0}^{2} e^{2}}}
$$

This equation gives the thickness of electron depletion layer of $70.8 \mathrm{~nm}$ for the parameters of Fig. 3. This is in agreement with the calculated value of $66.8 \mathrm{~nm}$.

As the ion acceleration scheme presented in this paper relies on the front side acceleration process, it is desired to confine the strongest electrostatic field at the target front surface or in its close vicinity. Consequently, the target thickness $d$ should be larger than $l$. Otherwise, the electrostatic pressure would not be able to balance the radiation pressure and the electron cloud will be fully expelled from the target volume. This will result in an acceleration of all ions with the strongest electrostatic field at the target rear side as it was described in Ref. [12]. However, we are not interested in this regime, as the acceleration process there cannot benefit from the ballistic evolution.

Ions start to respond to the strong electrostatic field in the second step of interaction as it was described in the model [14]. The charge-separation field in the electron depletion layer is not uniform. It is increasing with the distance from the initial target surface up to the position of the compressed electron cloud, where it attains its maximum given by Eq. (1). The velocity of ions in the electron depletion layer thus depends on their initial position. The velocity of ions in the compressed electron cloud can be found by equating the electrostatic pressure and the dynamic pressure

$$
\rho v_{i}^{2}=\varepsilon_{0} E_{\mathrm{es}}^{2}
$$

where $\rho=m_{i} n_{i}$ is the ion mass density and the dynamic pressure due to electrons has been neglected. It results from the equation of motion with the Lorentz force and Eq. (4) that the electrostatic field with amplitude $E_{\mathrm{es}}$ accelerates ions to the velocity $v_{i}$ during the time interval of $\tau_{2}=$ $1 / \omega_{p i}$ ( $\approx 1.5$ laser period for the parameters used in the PIC simulation); that is, the characteristic time scale of the second step is the inverse ion plasma frequency.

As a first approximation, let us assume that all ions in the compressed electron cloud gain the same velocity $v_{i}$. Then they will cross the electron cloud and form a shock structure propagating into the target with this velocity. For the sake of simplicity, let us further neglect any ion accelera- tion process taking place behind the compressed electron cloud. Then the population of ions deeper inside the target consists of three distinct groups: the stationary unperturbed ions, the ions accelerated inside the compressed electron cloud to the velocity $v_{i}$, and the ions originating from the electron depletion layer with the velocities ranging between $v_{i}$ and $2 v_{i}$.

The phase space of ions in the second stage is shown in Fig. 4(a) at the time of $4 \tau$ after the beginning of interaction. The gray color transition in the background depicts the strength of the electrostatic field versus the spatial position. The electrostatic field is strongest at $x=3.15 \lambda$, at the instantaneous position of the front of the compressed electron cloud. According to Eqs. (1) and (4), the velocity $v_{i}$ gained by ions in the compressed electron cloud should be approximately $0.05 c$. However, as the electrostatic field in the PIC simulation is slightly weaker than the one predicted
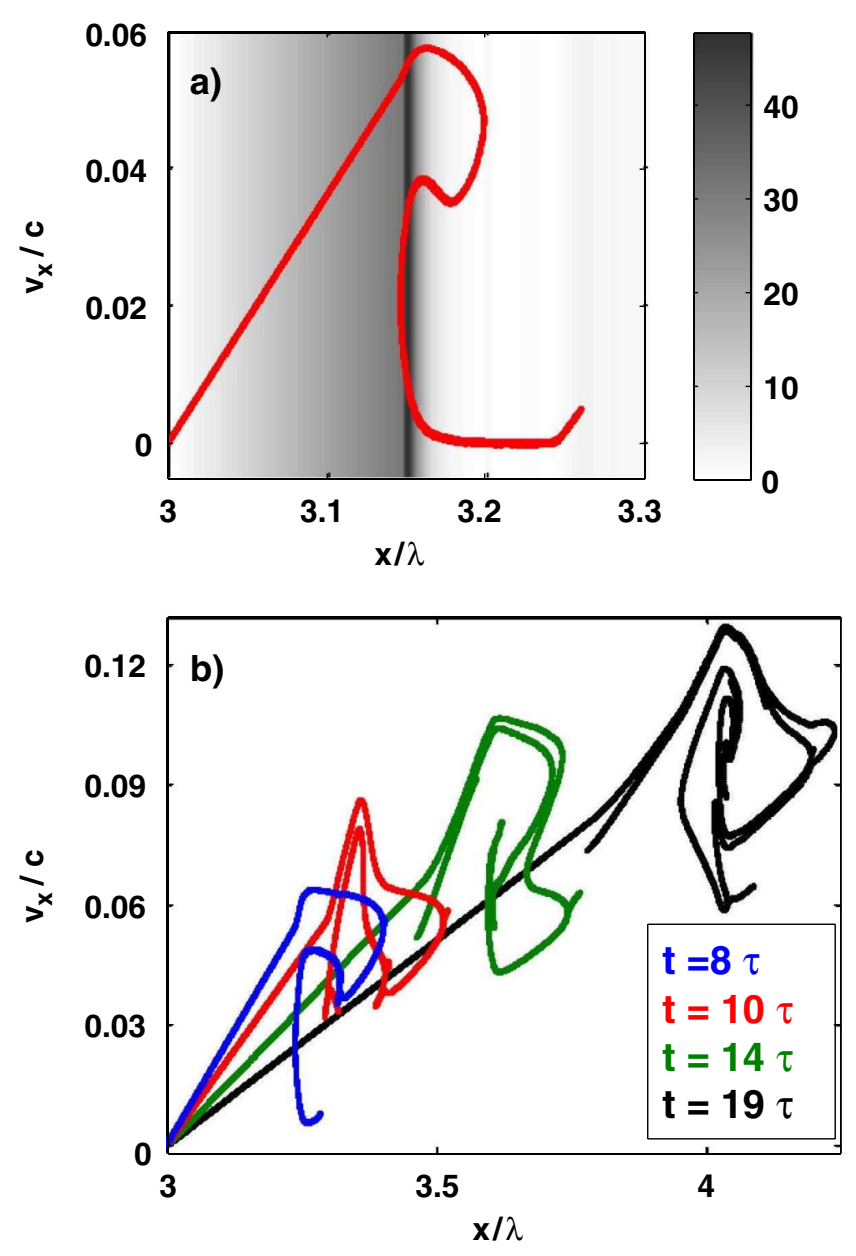

FIG. 4. (Color) (a) Ion phase space at the interaction time $4 \tau$. The gray color transition in the background denotes the actual strength of the electrostatic field in the units of $\mathrm{TV} / \mathrm{m}$ (see the colorbar for the scale). (b) Snapshots of the ion phase space at time $8,10,14$, and $18 \tau$. The loop originates from the combination of the front side acceleration and the ballistic evolution. The simulation parameters are the same as in Fig. 3. 
by Eq. (1), the velocity of ions accelerated in the electron cloud is lower, $0.038 c$. The ions with velocities higher than $v_{i}$ are originating from the electron depletion layer as discussed above. One can also distinguish acceleration of ions from the target rear side which was initially located at the spatial position of $3.25 \lambda$. This is in agreement with the structure of the electrostatic field observed in Fig. 3. However, this process is much weaker and it is neglected in what follows.

Up to this point, the theory and simulation results presented here are in accordance with the ion acceleration model of Macchi et al. [14]. However, we follow the acceleration of ions further. Once all ions in the compressed electron cloud are accelerated and enter the nonperturbed target, the electron cloud is pushed deeper into the target as well. It can move only a distance of the order of skin depth because there it encounters the next layer of stationary ions. These ions start to experience the strong electrostatic field and they are accelerated just like the ones before. The acceleration of ions thus proceeds layer by layer and because of the constant radiation pressure assumed here, more and more ions are gradually accelerated to the same velocity $v_{i}$. In reality, the acceleration process is not steplike but continuous. The inertia of electrons is much lower than that of ions. Therefore, the compressed electron cloud adjusts its position and density selfconsistently to the moving ions. The electrostatic potential at the front side of the target does not change, and the acceleration proceeds as a stationary wave.

This third step of ion acceleration lasts until the moment when the compressed electron cloud reaches the position of the former rear target surface. At that moment all ions are accelerated to nearly the same velocity $v_{i}$, Eq. (4). That gives us an opportunity to estimate the duration of this interaction stage, $\tau_{3}$, from the condition that the momentum of the whole target (per unit area) equals the radiation pressure exerted on the target surface during that time,

$$
\rho v_{i} d=2 I_{L} \tau_{3} / c .
$$

According to this expression, the third stage terminates at $10 \tau$ after the beginning of the interaction in our PIC simulation. This is confirmed by the temporal evolution of the ion phase space in Fig. 4(b), where all the ions from inside of the foil have already been accelerated to velocities higher than $v_{i}$ at the time $10 \tau$. The ions from the electron depletion layer are an exception. Some of these ions remain relatively slow and because of the electrostatic field shape they will not be able to catch the moving foil any more.

The fourth stage begins after the whole target has been accelerated. This stage is actually the most important one in the present context. The ballistic evolution of the accelerated ions plays an important role there. The slower particles are overtaken by the faster ones and, consequently, they appear on the actual target front side where they are strongly accelerated. In fact, the fourth stage can be characterized by a series of loops of the accelerated ion bunch in the phase space. The first of these loops is demonstrated in the temporal evolution of the ion phase space in Fig. 4(b). The ballistic evolution combined with the continuing front side acceleration naturally results in acceleration of all ions to a well-defined macroscopic propagation velocity. Moreover, the velocity spread of accelerated ions does not increase with time as can be also seen in Fig. 4(b). In consequence, the target volume is conserved and therefore it is possible to maintain the acceleration process in the same form for a long time. One may accelerate the ion bunch to a very high velocity comparable with the velocity of light. This is a big advantage in comparison with the TNSA mechanism, where the accelerating electrostatic field decreases with time.

The velocity of the ion bunch after the interaction, $v_{f}$, can be found from Eq. (5) by setting the time on the righthand side to the laser pulse duration $t_{L}$. Using this macroscopic approach, we neglect the laser absorption and the dispersion of ion velocities, which is justified by the fact that the target occupies a relatively small volume of the phase space during the interaction. The final velocity $v_{f}$ of the accelerated ion bunch calculated in this manner is about $0.152 c$. The average velocity of ions in the accelerated foil in the PIC simulation after the interaction is $0.149 c$, if the slower ions from the electron depletion layer are not included (the velocity averaged over all ions is $0.145 c$ ). The mean velocity dispersion in this example is about $0.014 c$, that is, less than $10 \%$.

From the final velocity of the ion bunch, $v_{f}$, one can calculate its kinetic energy, $\rho d v_{f}^{2} / 2$, and estimate the fraction of the laser beam energy, $\eta I_{L} t_{L}$, absorbed by ions as

$$
\eta=\frac{2 I_{L} t_{L}}{c^{2} \rho d}=\frac{v_{f}}{c} .
$$

It is obvious that this expression and all the estimates given above are valid only in the limit $v_{f} \ll c$. The theory can be generalized to include the relativistic mass of ions and the laser pulse absorption due to the Doppler shift of the reflected light. However, as will be demonstrated below, it does not seem to be realistic to accelerate the whole foil to a relativistic velocity where these corrections are necessary. The laser pulse energy absorption into ions is $14.3 \%$ in the present PIC simulation.

Equation (6) states that the efficiency of the acceleration process increases linearly with the laser pulse duration for a given laser intensity. Such a behavior is not common in short pulse laser-target interactions, and it results from the fact that the fourth stage of interaction is characterized by a direct momentum transfer from the laser pulse to the whole target. On the contrary, during the third stage of interaction the number of accelerated ions scales linearly with the time, while their maximum velocity remains constant. 
Then the efficiency of laser energy transformation, $\eta=$ $v_{i} / c$, is independent on time.

If the desired energy of monoenergetic ions is given, their momentum is also defined, assuming that these ions propagate only in the laser incidence direction. The maximum momentum that can be transferred to the target is $2 I_{L} t_{L} / c$. As this ion acceleration scheme relies purely on the momentum transfer from the laser pulse to the monoenergetic ion beam, it should be the most efficient ion acceleration mechanism in terms of the total energy contained in the monoenergetic bunch of ions. In all other ion acceleration schemes, a significant amount of momentum (energy) may be contained in the population of hot electrons or in the population of ions with different energies.

It can be seen in Fig. 5, that the velocity spread of the ion beam does not increase during the fourth stage of interaction. This spread is less than $2 v_{i}$ at the end of the third stage, and the majority of accelerated ions have their velocities in the range $v_{f} \pm v_{i}$ after the interaction. For the case of simulation presented here, the ratio between $v_{f}$ and $v_{i}$ is only 3.8. By increasing the laser pulse duration, one can increase the final velocity $v_{f}$ while keeping the same velocity $v_{i}$. This opens a possibility to control the energy spread of ions through the foil thickness and the laser pulse duration.

For example, to keep the number density and the energy of the accelerated ions constant, one should prescribe the foil areal density and the laser pulse energy. Then the interaction of a longer laser pulse with a thinner foil should provide the ion beam with a lower energy spread. This is demonstrated in the PIC simulation with the 2 times longer laser pulse and 2 times denser foil in Fig. 5. According to

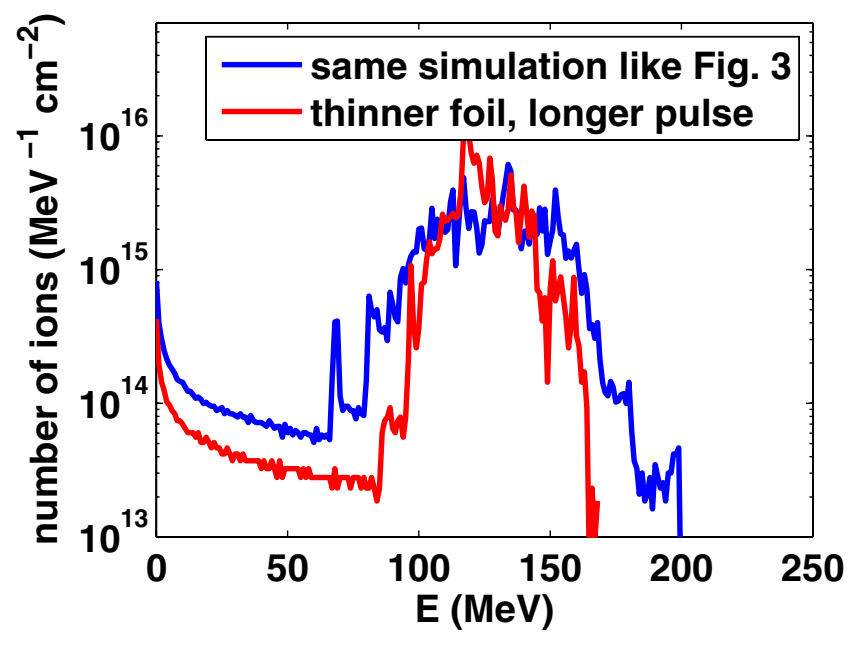

FIG. 5. (Color) Energy distributions of ions at the end of the laser foil interaction. The wider distribution (blue) corresponds to the simulation parameters given in Fig. 3, the narrower distribution (red) corresponds to the simulation where the foil is 2 times thinner and 2 times denser and the laser pulse is 2 times longer and 2 times less intense.
Eq. (4), the initial velocity spread of ions is decreased 2 times in this case and thus the width of the energy distribution is reduced to one-half of the energy distribution in the former case.

The fifth stage is the last one. It covers the target evolution after the end of the laser pulse. The temperature of electrons increases immediately after the radiation pressure is removed. Then the electrostatic field accelerates electrons across the front boundary of the target and they are gaining the energy $\epsilon_{e} \sim 2 I_{L} / c n_{e 0} \approx 3 \mathrm{MeV}$. As the ion bunch still occupies a relatively small volume, the accelerated electrons start to circulate across the bunch creating electrostatic fields which accelerate ions from both surfaces of the target. Nevertheless, if the fourth stage of interaction is sufficiently long, the kinetic energy of ions, $\sim \frac{1}{2} m_{i} v_{f}^{2}$, is significantly higher than the kinetic energy of accelerated electrons, $\epsilon_{e}$, and, therefore, the electrons should not broaden much the energy spectrum of ions. The electrons are gradually cooling, whereas the dense bunch of ions evolves also ballistically. Thus, in the later time, the electron Debye length becomes smaller than the dimension of the bunch and the Coulomb repulsion becomes insignificant.

The crucial point of the ion acceleration approach discussed here is that the overall quasineutrality of the target is maintained until the end of the laser-target interaction. Accordingly, the Coulomb repulsion takes place only at the late stage of the process and plays a much less important role than in the TNSA. It is possible to maintain a narrow ion spectrum until they hit a detector or a secondary target.

A good one-dimensional stability of the ion acceleration process and reliability of our analytical estimates have been tested in PIC simulations covering a wide range of interaction parameters. The laser pulse intensity has been varied in the range between $3 \times 10^{19}$ and $3 \times$ $10^{20} \mathrm{~W} / \mathrm{cm}^{2}$, the laser pulse duration between 40 and $400 \mathrm{fs}$, the foil thickness between 30 and $300 \mathrm{~nm}$, and the foil mass density between 0.08 and $0.8 \mathrm{~g} / \mathrm{cm}^{3}$. The acceleration process remains stable even if the density profile on the foil surface is not steplike but steep, with a relatively short scale length $L<0.1 \lambda$. The process described above should theoretically be stable whenever the radiation pressure is much higher than the thermal pressure and the electron heating is inhibited so that the ion acceleration process takes place, in particular, on the target front side.

\section{Targets composed of multiple species of ions}

Because of their highest charge to mass ratio, protons are accelerated with a higher efficiency than other heavier ions in most ion acceleration experiments. Even if the target material does not contain protons, they are usually present in thin layers of contaminants on the target surface. These contaminants have to be removed in order to accelerate higher $Z$ ions efficiently with the TNSA process. However, 
in the case of the ponderomotive ion acceleration, the light ions do not radically inhibit the acceleration efficiency of heavier ions and thus the contamination of the target surface does not pose a serious problem.

At the first sight, it might be surprising, but there is no charge state dependence in the theory given above. In fact, the only two parameters depend on the charge state of ions. These are the thickness of the electron depletion layer (through the dependence on $n_{e 0}=Z n_{i 0}$ ) and the ion plasma frequency. For example, with a lower charge state, the ion plasma frequency is shorter and the ion acceleration takes a longer time. On the other hand, the thickness of the electron depletion layer is larger in this case and thus more ions are accelerated at the same moment. These two effects cancel each other and, consequently, the charge state dependence is removed from the theory. Therefore, in addition to a narrow energy spread in the bunch of accelerated
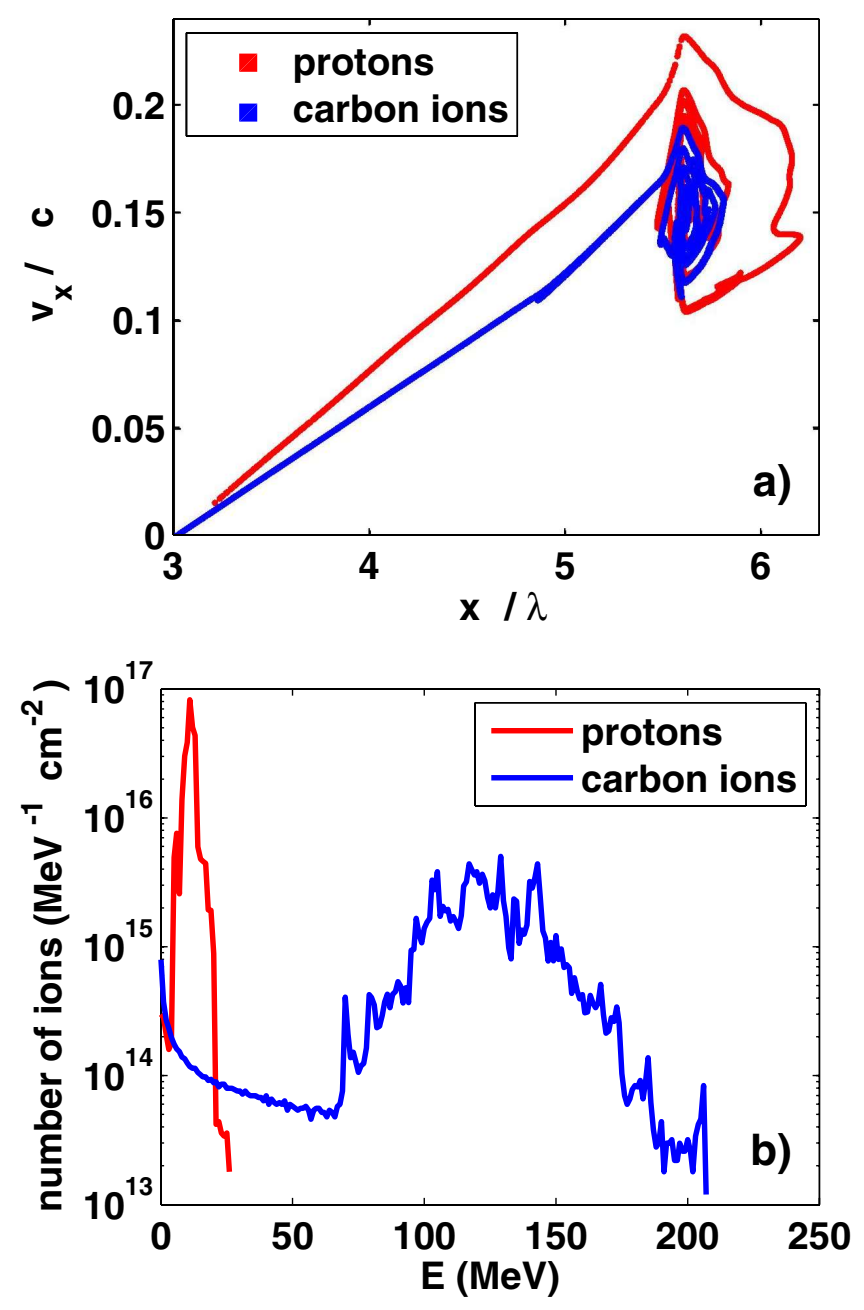

FIG. 6. (Color) Ion phase space (a) and ion energy distributions (b) at the end of the interaction of the laser pulse with a foil composed of fully ionized carbon ions and protons. The density of carbon ions is $7.5 \times 10^{21} \mathrm{~cm}^{-3}$ and the density of protons is 2 times higher. The other simulation parameters are the same as in Fig. 3 (the mass density of the foil is the same as well). ions, the presented ion acceleration process is also advantageous in the sense that all ions are accelerated to the same velocity irrespectively to their charge to mass ratio. Moreover, due to the ballistic evolution of the target during the acceleration process, all ions acquire the same velocity even in the targets composed of multiple species. This means that higher $Z$ ions may attain much higher energy than protons without the need of removing any proton rich contaminants from the target surface.

For demonstration, the results of ion acceleration in 1D3V PIC simulation with a multispecies target are presented in Fig. 6. The parameters of the laser pulse and the foil are the same as in the previous subsection with the exception of the material composition. The foil now contains the carbon ions with the density of $7.5 \times 10^{21} \mathrm{~cm}^{-3}$ and protons with the density of $1.5 \times 10^{22} \mathrm{~cm}^{-3}$ (a low density plastic foil $\mathrm{CH}_{2}$ ). The mass density of the foil is not changed. The ion phase space at the end of the interaction plotted in Fig. 6(a) demonstrates that both ion species are accelerated to a nearly same velocity. To be exact, the average velocity of carbon ions is $0.143 c$ and the average velocity of protons is $0.155 \mathrm{c}$. A higher charge to mass ratio of protons results in their stronger acceleration (higher $v_{i}$ ) in the electrostatic field which is confined on the target front side by the heavier carbon ions. Therefore, some protons propagate ahead of the beam of carbon ions and the beam of accelerated protons has also slightly higher velocity spread. However, in spite of a higher number density of protons, the efficiency of acceleration of carbon ions is not decreased significantly and their narrow energy distribution is not affected, as can be seen in Fig. 6(b).

Multispecies targets may even be advantageous in this ion acceleration process as in the fifth stage after the end of laser pulse, the protons help to confine the narrow spectrum of carbon ions. The response of protons to the electrostatic field is faster and thus they, at least partially, screen the field produced by fast electrons on the foil surface.

\section{E. Two-dimensional simulations}

The presented ion acceleration process may be sustained in nearly the same form for a long time in one spatial dimension and it seems possible to accelerate ion beams to relativistic velocities. However, multiple spatial dimensions bring some additional constraints, which might be difficult to overcome. Let us consider the same laser intensity $\left(3 \times 10^{20} \mathrm{~W} / \mathrm{cm}^{2}\right)$, the same foil thickness $(200 \mathrm{~nm})$, and the same foil mass density $\left(0.176 \mathrm{~g} / \mathrm{cm}^{3}\right)$ like in the previous subsection. According to Eq. (5), the protons will be accelerated to $1 \mathrm{GeV}$ with the laser pulse duration of $460 \mathrm{fs}$. In fact, the pulse would have to be even longer if one accounts for the relativistic mass correction and the finite reflectivity. As the acceleration force is constant in time, one easily calculates the distance, $\sim 60 \mu \mathrm{m}$, which the foil moves during the acceleration process. This distance is typically longer than the 
Rayleigh length of the focused laser pulse. Therefore, one has to account for the laser pulse divergence or a laser pulse guiding has to be considered. The radial dependence of the laser intensity may also increase the energy spread in the ion bunch, according to Eq. (5).

Another constraint comes from the finite size of the laser focal spot on the target. Because of the radial dependence of the laser intensity, the foil feels spatially dependent acceleration force and its surface becomes curved during the interaction. Once the curvature of the foil surface becomes significant, the local angle of incidence of the laser beam may provoke an enhanced absorption and a strong electron heating in certain spatial regions. The foil shape $s(r)$ at the time $t<t_{L}$ can be calculated in the same manner as above, supposing that the laser intensity radial distribution is known:

$$
s=\frac{I_{L}(r)}{2 c \rho d} t^{2} .
$$

The incidence angle of the laser pulse is $\sim 45^{\circ}$ at the points where $d s / d r \sim 1$. At the moment when this condition is verified, the electron heating becomes efficient and the surface of the hole created in the foil starts to feel a strong acceleration force in the radial direction. This is the result of the ponderomotive force of the laser pulse, which is reflected from the lateral surface of the hole inward the hole. Let us suppose that the laser beam has a Gaussian intensity profile, $I_{L}(r)$, with the full width at half maximum $\sigma$. Then from Eq. (7) we estimate the time interval, where the presented ion acceleration model is applicable, as

$$
t_{\max } \simeq \sqrt{\sigma c \rho d / I_{0}} .
$$

For the parameters considered above and for $\sigma=10 \mu \mathrm{m}$, the maximum acceleration time is about $200 \mathrm{fs}$ and the maximum velocity and energy of the monoenergetic beam of protons are about $0.36 c$ and $70 \mathrm{MeV}$, respectively. The ratio between the duration $\tau_{3}$ of the first three stages of the acceleration process and $t_{\text {max }}$ depends on the ratio between the focal spot size and the target thickness. One has to use relatively large focal spots with a flattop intensity profile for acceleration to high ion energies, which makes this acceleration process more complicated with respect to the experimental feasibility. It is also possible to increase $t_{\max }$ by shaping the foil or by using the foil with spatially dependent thickness or mass density. However, it would be difficult to realize such an optimization in experiment because of the pointing accuracy of the laser beam. One may also think of utilizing an ultrasmall foil with spatial dimensions smaller than the laser focal spot. However, fast electrons would be efficiently produced on the borders of such a foil and the ion acceleration process will be significantly affected [26].

Nevertheless, as the above constraints come from the motion of the foil, the limiting factor is the velocity of ions, not their energy. Therefore, the ion acceleration process described in this paper is better suited for acceleration of heavier ions, which may attain kinetic energies in the hundred $\mathrm{MeV}$ range.

The effects of the radial intensity distribution on the ion acceleration were studied with our 2D3V PIC code. The laser pulse has a flattop intensity profile on the target surface as shown in Fig. 7. It has a Gaussian spatial intensity profile with $\sigma=2.9 \mu \mathrm{m}$ at the edges and a $5 \mu \mathrm{m}$ intensity plateau in the central part. The carbon foil is $14 \mu \mathrm{m}$ wide in the transverse dimension and other parameters are the same as in the 1D simulation presented in Sec. IIIC. The spatial density profile of ions in the simulation box is shown in Fig. 8 at two time instants. The ion density is in units of the critical density $(1.75 \times$ $10^{21} \mathrm{~cm}^{-3}$ ). In panel (a), the ion density is plotted in the time $8 \tau$ which is slightly before the end of the third ion acceleration stage. The imprint of the laser intensity profile on the density of ions is clearly seen. The interaction time is $28 \tau$ in panel (b) and the hole in the center of the foil is observed. The time $28 \tau$ is still smaller than $t_{\max }$, which is $37 \tau$ for our simulation parameters. The laser incidence angle onto the lateral borders of the hole is about $35^{\circ}$.

The electric field $E_{x}$, pointing in the laser propagation direction, is plotted in Fig. 9. At the time $8 \tau$ [panel (a)], one sees the front side electrostatic field which is accelerating the ions. The longitudinal electric field of the laser wave which is due to the finite focal spot can be seen in front of the target. This field may be responsible for enhanced electron heating if the laser beam is tightly focused [27]. Nevertheless, this electron heating does not play an important role in the simulations of the ion acceleration process presented here. The structure of the electric field $E_{x}$ is more complex in panel (b) at $28 \tau$. It contains the front

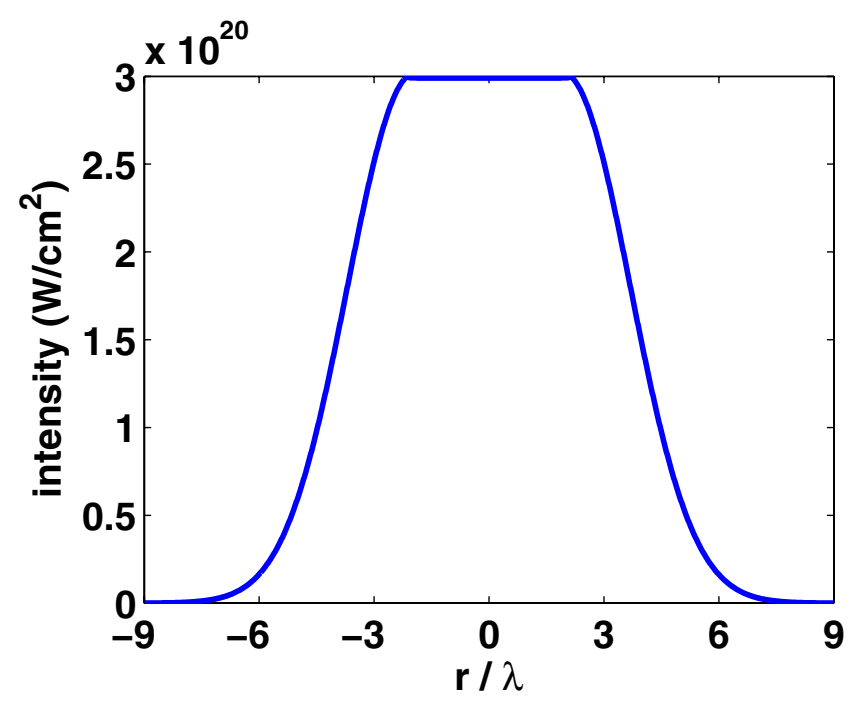

FIG. 7. (Color) Spatial laser intensity profile used in 2D3V PIC simulations. This profile has $5 \mu \mathrm{m}$ plateau in the central part and it is Gaussian on both sides of the plateau (with the characteristic scale of $2.9 \mu \mathrm{m})$. 

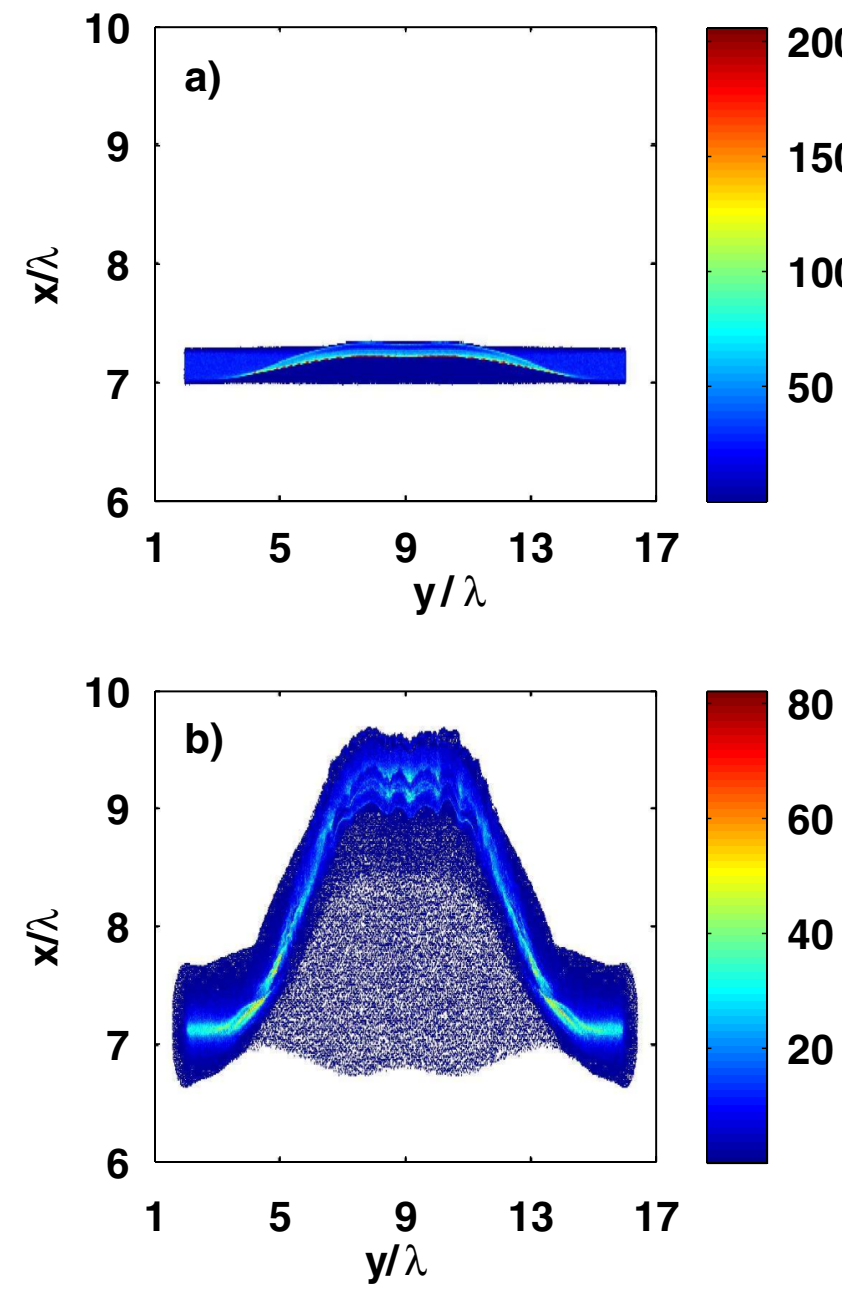

FIG. 8. (Color) Spatial ion density profile in the 2D3V PIC simulation in the interaction time $8 \tau$ (a) and $28 \tau$ (b). In the interaction time $28 \tau$ a hole bored in the central part of the foil can be observed. The simulation parameters are the same as in Fig. 3 with the radial intensity profile of the laser pulse shown in Fig. 7. The ion density is normalized to the critical density and the spatial coordinates are normalized to the laser wavelength. The midpoint of the simulation box is at $9 \lambda$ and the laser pulse is incident from the bottom.

side electrostatic field and also the field on the rear side of the foil, which is due to already accelerated fast electrons. Moreover, the laser field reflected from the lateral borders of the hole is clearly visible in the central part. As this field is almost as strong as the field of the incident laser pulse, the radiation pressure strongly pushes the borders of the hole in the transverse direction as well.

The electrostatic field on the front surface of the foil in Fig. 9(b) exhibits a regular structure in the central part. This structure corresponds to the ion density fluctuations observed in the same spatial position in Fig. 8(b). The density fluctuations are probably resulting from the radiation dominated Rayleigh-Taylor instability, which was analyzed in Refs. [10,28]. The growth rate of this instability is
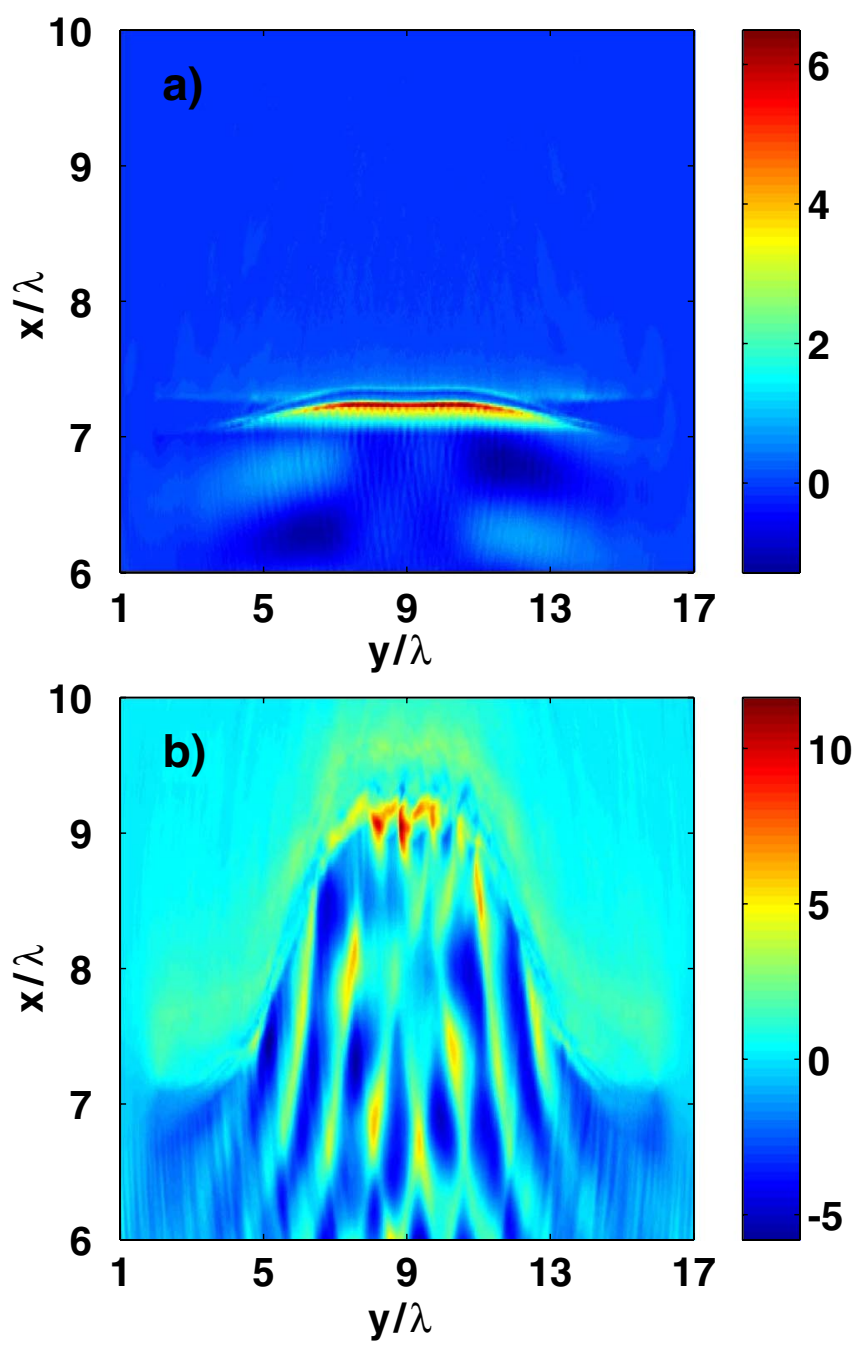

FIG. 9. (Color) Electric field in the laser incidence direction, $E_{x}$, in the normalized units $\left(m_{e} c \omega / e\right)$. The simulation parameters and time instants are the same as in Fig. 8. At the interaction time $28 \tau$ the field in the central part contains also the specularly reflected part of the laser wave.

$$
\gamma=\sqrt{k_{y} v_{i}^{2} / 3 l_{s}}
$$

where $k_{y}$ is the wave number of the unstable mode, $v_{i}$ is the ion velocity given by Eq. (4) (in [28] denoted as the Alfvén velocity), and $l_{s}$ is the collisionless skin depth. The wave number of density fluctuations observed in Fig. 8(b) is about $4 \pi / \lambda$ and for the conditions of our simulations, the growth time of this mode (9) should be $\sim 2 \tau$. This is approximately the same as the characteristic response time of ions to the electrostatic field $\left(1 / \omega_{i}\right)$ and the ion density corrugations become visible in the interaction time of about $25 \tau$.

The phase space of ions plotted in Fig. 10(a) provides a clear evidence that the ion acceleration process in the central part of the foil works in the same way as in the 1D simulation. Only the ions located in the $2 \lambda$ wide central 


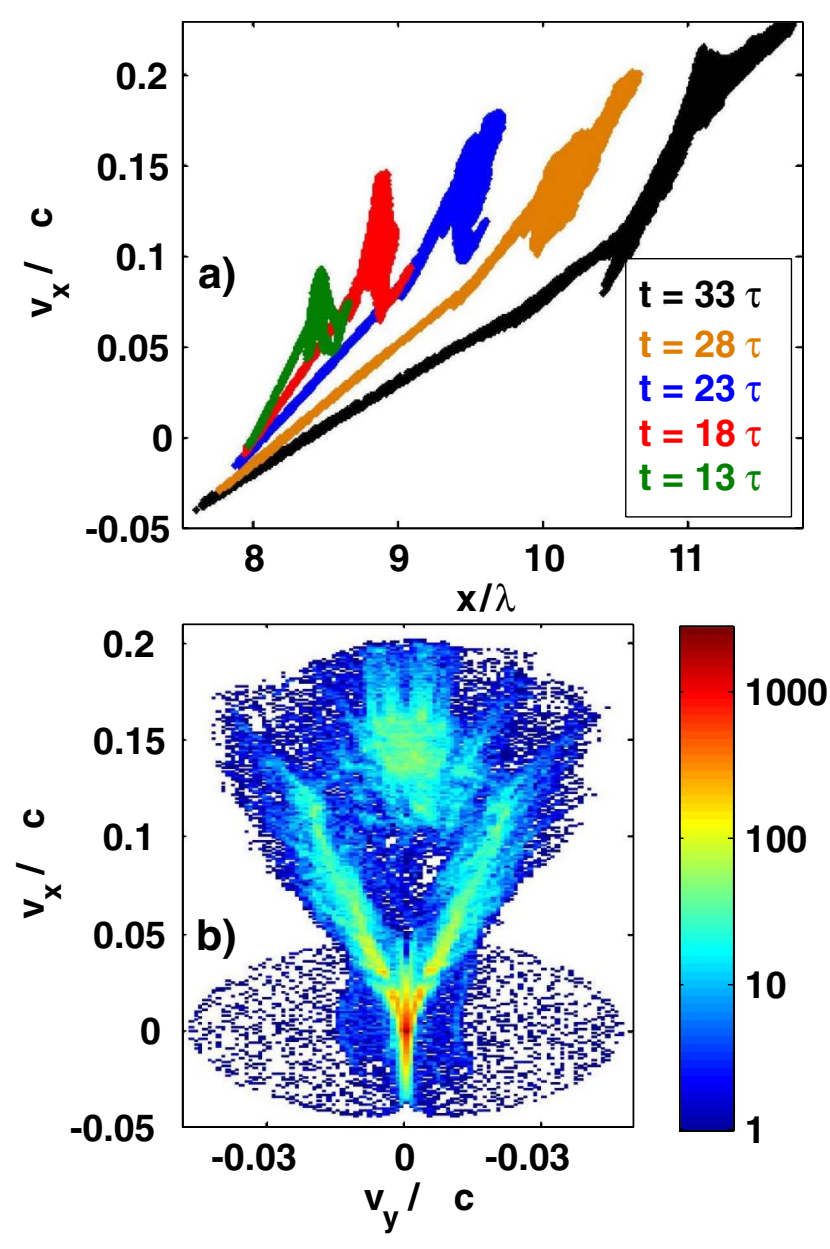

FIG. 10. (Color) Temporal evolution of the ion phase space [panel (a)] and the ion velocity distribution in the interaction time $28 \tau$ [panel (b)] in the 2D3V PIC simulation. The parameters are the same as in Fig. 8. The ion phase space contains only ions located in the $2 \lambda$ wide central part of the simulation box. All ions are included in the velocity distribution in panel (b), where the color scale is logarithmic and the number of ions is in arbitrary units.

region $(8 \lambda \leq y \leq 10 \lambda)$ are included in this figure. It can be seen that, up to the interaction time of $28 \tau$, most of the ions are contained in a single dense bunch, which has a welldefined macroscopic velocity and a relatively low velocity spread. However, the bunch of ions is significantly dispersed at the time $33 \tau$. Therefore, the interaction should be terminated before the time of about $30 \tau$ to preserve a narrow spectrum of accelerated ions. Thus, the limiting time given by Eq. (8), which is about $37 \tau$ in this case, is slightly overestimated.

The distribution function plotted in Fig. 10(b) at the time of $28 \tau$ contains all ions. The monoenergetic bunch accelerated in the central part of the foil can be recognized here. As the bunch propagates with the velocity of about $0.15 c$ in the $x$ direction and its velocity spread in the $y$ direction is lower than $0.02 c$, this bunch of ions is relatively well collimated. The transversely expanding borders of the hole created in the foil are also clearly observed. They form a conical jet with the opening angle of about $12^{\circ}$, which is related with the radial distribution of the laser beam intensity. In fact, this is a good sign as it indicates that the slower ions with a broad energy spectrum form a divergent ring structure around the central collimated monoenergetic bunch and thus the slower ions may be easily filtered out. One can also see a significant population of ions which are located outside the focal region and therefore they remain relatively cold.

The energy distributions of ions and electrons in the central part of the foil $(8 \lambda \leq y \leq 10 \lambda)$ are plotted in Fig. 11. The energy distribution of ions in panel (a) exhibits a peak structure, which significantly broadens in time (compare the curves corresponding to the simulation times $28 \tau$ and $33 \tau$ ). However, the peak is clearly visible all the interaction time $(33 \tau)$. The broadening is due to interaction of ions with the field produced by fast electrons, which are efficiently accelerated in the later stage of the interaction process. To verify that the ion energy distribution remains quasimonoenergetic after the end of the laser-target interaction, we have performed an additional simulation run with a shorter laser pulse (duration of $20 \tau$ ). The energy distribution of ions resulting at the end $(38 \tau)$ of this simulation is included in Fig. 11(a). This energy distribution did not change significantly during the last $5 \tau$ in the simulation which confirms that the strongest ion acceleration process is over at this time instant.

For comparison, the energy distribution of ions resulting at the end of 1D simulation is also included. The peak from the 1D simulation is narrower, but the difference is in the edges. It is also remarkable that the ions in the 2D simulation attain the same energy earlier than in the 1D simulation, and the final energy of the ion bunch is significantly higher. This feature is again connected to the fast electrons, which contribute not only to the energy spread of accelerated ions but they also increase the average energy of the ion bunch.

The conversion efficiency of the laser pulse energy into the quasimonoenergetic ion bunch accelerated from the central part of the foil $(8 \lambda \leq y \leq 10 \lambda)$ in the 2D simulation with the $20 \tau$ long laser pulse is $5.5 \%$. To confirm that acceleration of ions to very high energies is enhanced by using the circularly polarized laser wave, we have also performed 2D simulation with the same parameters but with a linearly polarized laser pulse. The conversion efficiency of laser energy into all ions in the simulation box with energy higher than $50 \mathrm{MeV}$ is $12.2 \%$ in the case of circular polarization and only $2.5 \%$ in the case of linear polarization.

The temporal evolution of the electron energy spectra in the $2 \mathrm{D}$ simulation with the longer $(30 \tau)$ laser pulse is plotted in Fig. 11(b). In the first part of the interaction $(t \lesssim$ $23 \tau$ ), the temperature of electrons is relatively low, below 

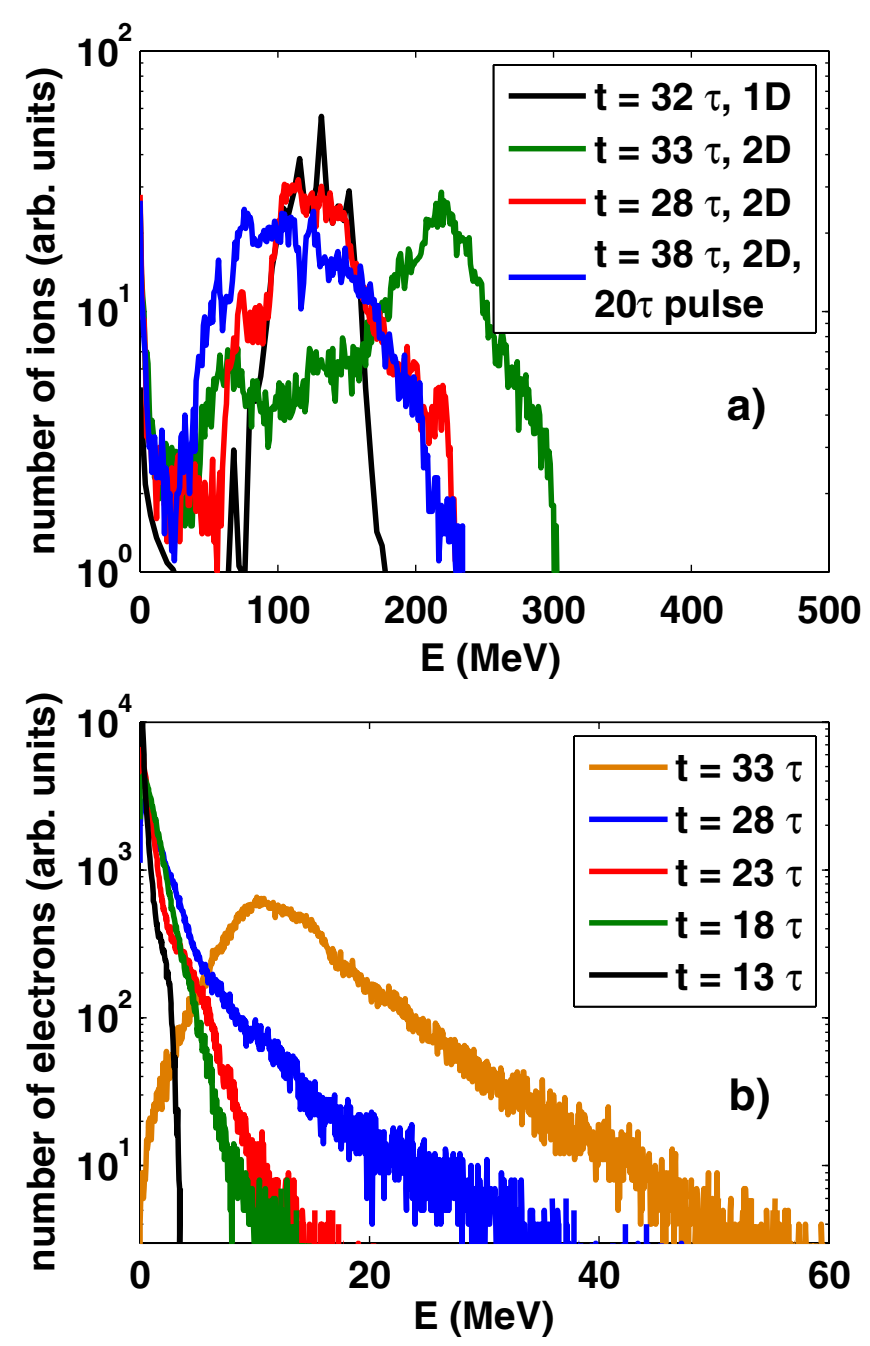

FIG. 11. (Color) The energy distributions of ions [panel (a)] and electrons [panel (b)] in the $2 \lambda$ wide central part of the simulation box in the 2D3V PIC simulation with the parameters of Fig. 8. The distribution from 1D3V PIC simulation with the same parameters is included for comparison in panel (a). The energy distribution of ions resulting from the 2D3V PIC simulation with a shorter (20 $\tau$ long) laser pulse is plotted in the time $18 \tau$ after the end of the laser-target interaction to demonstrate that the quasimonoenergetic distribution is preserved. The energy distribution of electrons in panel (b) is peaked at very high energy in the time $33 \tau$. This is the consequence of the explosion of the central part of the foil which became underdense to the laser pulse before this time.

1.9 MeV. However, as soon as the surface of the foil gets curved significantly, the temperature of electrons strongly rises and it is already about 3 times higher at the time of $28 \tau$. As noted above, the interaction should be terminated before this time to maintain a narrow energy spread in the bunch of accelerated ions as in the case of the shorter $(20 \tau$ long) laser pulse. As a consequence of electron heating, the target starts to explode and it becomes transparent to the relativistic laser pulse at the end of the run $(33 \tau)$. The energy of electrons raises to about $10 \mathrm{MeV}$.
The limitations imposed on acceleration by laser beam edges could be relaxed significantly, if the target ions are much heavier than the carbon ions considered here.

\section{SUMMARY AND CONCLUSIONS}

In this paper, we propose a new mechanism of a monoenergetic ion beam generation that is especially suitable for acceleration of heavy ions where the existing ion acceleration mechanisms are inefficient. This mechanism is based on the interaction of a short intense circularly polarized laser pulse with an ultrathin overdense foil. It is demonstrated that this interaction may result in acceleration of a single dense collimated bunch of ions, which is appropriate for many applications. The formation of a monoenergetic ion spectrum results from a combination of acceleration of ions in the electrostatic field on the front side of the target with the ballistic evolution of the target itself. The unwanted electron heating is suppressed by using the circular laser polarization, a high intensity contrast, and the normal incidence.

The process of formation of a monoenergetic ion bunch proceeds in four subsequent stages: (i) an adiabatic compression of electrons in a thin layer on the front surface of the target, (ii) an acceleration of ions on the front surface of the target in the electron depletion layer and the compressed electron cloud, (iii) a gradual acceleration of all ions contained in the foil, and (iv) a further acceleration of the propagating ion bunch which itself evolves ballistically.

The ion acceleration process is described by a simple analytical theory, which is successfully compared with the results of our one-dimensional PIC simulation model. It is found that the ponderomotive force of a circularly polarized laser wave accelerates the whole ultrathin foil as a compact dense block of quasineutral plasma, which propagates with a well-defined macroscopic velocity. The acceleration process is thus well described by the momentum transfer from the laser pulse to the foil and the efficiency of this process scales with the foil velocity. It may be relatively high and the energy contained in the beam of monoenergetic ions is probably the highest possible of all ion acceleration processes because the ion energy spectrum is formed directly by the laser ponderomotive force.

In the final stage of acceleration, the ballistic evolution of the foil allows for the slower ions to be more strongly accelerated and the macroscopic velocity of the foil increases, while the velocity spread of ions contained in the foil remains constant. The velocity spread of ions in the beginning of this stage is related to the foil density and the laser pulse intensity, and it can be controlled through these parameters. It is also found that during this stage all species of ions attain nearly the same velocity irrespective of their charge to mass ratio. This means that a contamination of the target by lighter ions (protons) is not a hinderance in the acceleration process as both species of ions attain the same velocity and the heavier ions absorb more energy. 
The ion acceleration process becomes more complex and less efficient in multiple spatial dimensions. The main obstacle is curving of the front surface of the foil under the radial profile of the laser pulse intensity. To obtain a quasimonoenergetic and well collimated beam of ions, the laser pulse must have a flattop intensity distribution in the focal spot and the interaction must be terminated before the hole bored in the foil becomes too deep and the electron heating starts to be efficient. It is possible to obtain bunches of quasimonoenergetic ions with energies of tens $\mathrm{MeV}$ per nucleon. As the constraints on the interaction time in multiple dimensions come from the displacement of the central part of the foil, the limiting factors are connected with the velocity of the ion bunch. By using foils composed of heavier ions, one may attain energies in the hundred $\mathrm{MeV}$ range per nucleon. Such ion beams may be especially interesting in nuclear physics experiments.

In two-dimensional PIC simulations, the ion density corrugations caused by the radiation dominated Rayleigh-Taylor (RT) instability are seen at the later stage of the interaction. Such density corrugations may increase the efficiency of electron heating, which may cause an explosion of the quasimonoenergetic ion bunch in both the longitudinal and the transverse dimensions. However, our 2D simulation results indicate that the radial intensity profile of the laser pulse influences the ion acceleration process in the later stage more strongly than the RT instability, and the laser beam intensity distribution poses stronger constraints on the stability of the ion acceleration process.

\section{ACKNOWLEDGMENTS}

This work was supported by the Czech Ministry of Education, Youth and Sports under Project No. LC528 and by the Czech Science Foundation under Project No. 202/06/0801. The authors are grateful to John von Neumann Institute for computing in Juelich, Germany, for providing the computational resources, Project No. ECZ040.

Note added. - At this point, we also have to refer to two very recent papers of Zhang et al. [29] and of Robinson et al. [30], which came to our attention after this paper had been accepted and where acceleration of ions from ultrathin foils by circularly polarized laser pulse had been studied for similar conditions like in this paper. Our theory and results are therefore partially similar and partially complementary to those of $[29,30]$.

[1] S. P. Hatchett et al., Phys. Plasmas 7, 2076 (2000).

[2] A. Maksimchuk, S. Gu, K. Flippo, D. Umstadter, and V. Y. Bychenkov, Phys. Rev. Lett. 84, 4108 (2000).

[3] R. A. Snavely et al., Phys. Rev. Lett. 85, 2945 (2000).
[4] B. M. Hegelich, B. J. Albright, J. Cobble, K. Flippo, S. Letzring, M. Paffett, H. Ruhl, J. Schreiber, R. K. Schulze, and J. C. Fernandez, Nature (London) 439, 441 (2006).

[5] S.C. Wilks, A.B. Langdon, T.E. Cowan, M. Roth, M. Singh, S. Hatchett, M. H. Key, D. Pennington, A. MacKinnon, and R.A. Snavely, Phys. Plasmas 8, 542 (2001).

[6] J. F. M. Borghesi and O. Willi, J. Phys.: Conf. Ser. 58, 74 (2007).

[7] M. Zepf et al., Phys. Rev. Lett. 90, 064801 (2003).

[8] S. Ter-Avetisyan, M. Schnurer, P. V. Nickles, M. Kalashnikov, E. Risse, T. Sokollik, W. Sandner, A. Andreev, and V. Tikhonchuk, Phys. Rev. Lett. 96, 145006 (2006).

[9] A. V. Brantov, V.T. Tikhonchuk, O. Klimo, D. V. Romanov, S. Ter-Avetisyan, M. Schnurer, T. Sokollik, and P. V. Nickles, Phys. Plasmas 13, 122705 (2006).

[10] F. Pegoraro and S. V. Bulanov, Phys. Rev. Lett. 99, 065002 (2007).

[11] J. Denavit, Phys. Rev. Lett. 69, 3052 (1992).

[12] T. Esirkepov, M. Borghesi, S. V. Bulanov, G. Mourou, and T. Tajima, Phys. Rev. Lett. 92, 175003 (2004).

[13] L. Yin, B. J. Albright, B. M. Hegelich, K. J. Bowers, K. A. Flippo, T. J. T. Kwan, and J. C. Fernandez, Phys. Plasmas 14, 056706 (2007).

[14] A. Macchi, F. Cattani, T. V. Liseykina, and F. Cornolti, Phys. Rev. Lett. 94, 165003 (2005).

[15] M. Kado et al., Laser Part. Beams 24, 117 (2006).

[16] O. Klimo, J. Limpouch, and N. Zhavoronkov, J. Phys. IV 133, 1181 (2006).

[17] R. Lichters, R.E. W. Pfund, and J. Meyer-ter Vehn, Technical Report No. 225, Max-Planck Institute für Quantenoptik, Garching, 1997.

[18] J. Psikal, J. Limpouch, S. Kawata, and A. A. Andreev, Czech. J. Phys. 56, B515 (2006).

[19] I. Umeda, Y. Omura, T. Tominaga, and H. Matsumoto, Comput. Phys. Commun. 156, 73 (2003).

[20] T. Umeda, Y. Omura, and H. Matsumoto, Comput. Phys. Commun. 137, 286 (2001).

[21] Y. Sentoku, T.E. Cowan, A. Kemp, and H. Ruhl, Phys. Plasmas 10, 2009 (2003).

[22] D. Neely, P. Foster, A. Robinson, F. Lindau, O. Lundh, A. Persson, C. G. Wahlstrom, and P. McKenna, Appl. Phys. Lett. 89, 021502 (2006).

[23] T. Ceccotti, A. Lévy, H. Popescu, F. Réau, P. D’Oliveira, P. Monot, J. P. Geindre, E. Lefebvre and P. Martin, Phys. Rev. Lett. 99, 185002 (2007).

[24] T. V. Liseikina and A. Macchi, Appl. Phys. Lett. 91, 171502 (2007).

[25] B. F. Shen and J. Meyer-ter Vehn, Phys. Plasmas 8, 1003 (2001).

[26] F. He, H. Xu, Y. W. Tian, W. Yu, P.X. Lu, and R. X. Li, Phys. Plasmas 13, 073102 (2006).

[27] V. I. Berezhiani and N. L. Shatashvili, Europhys. Lett. 76, 70 (2006).

[28] E. G. Gamaly, Phys. Rev. E 48, 2924 (1993).

[29] X. Zhang, B. Shen, X. Li, Z. Jin, F. Wang, and M. Wen, Phys. Plasmas 14, 123108 (2007).

[30] A.P. L. Robinson, M. Zepf, S. Kar, R. G. Evans, and C. Bellei, New J. Phys. 10, 013021 (2008). 